\title{
Simulation of Evacuation Characteristics Using a 2-Dimensional Cellular Automata Model for Pedestrian Dynamics
}

\author{
Liqiang Ji, Yongsheng Qian, Junwei Zeng, Min Wang, Dejie Xu, Yan Yan, and Shuo Feng \\ School of Traffic and Transportation, Lanzhou JiaoTong University, Lanzhou, Gansu 730070, China \\ Correspondence should be addressed to Yongsheng Qian; qianyongsheng@mail.lzjtu.cn
}

Received 3 June 2013; Revised 4 August 2013; Accepted 4 August 2013

Academic Editor: Mohamed Fathy El-Amin

Copyright (C) 2013 Liqiang Ji et al. This is an open access article distributed under the Creative Commons Attribution License, which permits unrestricted use, distribution, and reproduction in any medium, provided the original work is properly cited.

\begin{abstract}
In public places, the high pedestrian density is one of the direct causes leading to crowding and trample disaster, so it is very necessary to investigate the collective and evacuation characteristics for pedestrian movement. In the occupants' evacuation process, the people-people interaction and the people-environment interaction are sufficiently considered in this paper, which have been divided into the exit attraction, the repulsion force between people, the friction between people, the repulsion force between human and barrier, and the attraction of surrounding people. Through analyzing the existing models, a new occupant evacuation cellular automata (CA) model based on the social force model is presented, which overcomes the shortage of the high density crowd simulation and combines the advantages that CA has sample rules and faster calculating speed. The simulating result shows a great applicability for evacuation under the high density crowd condition, and the segregation phenomena have also been found in the bidirectional pedestrian flow. Besides these, setting isolated belt near the exit or entrance of underpass not only remarkably decreases the density and the risk of tramper disaster but also increases the evacuation efficiency, so it provides a new idea for infrastructure design about the exits and entrances.
\end{abstract}

\section{Introduction}

In recent years, the large public activities have become very common, especially the large public collection that leads to severe clogging and crowding, which brings high risk of happening trample incidents and may result in huge casualty and property losses. According to the statistics, too many large-scale crowd trample disasters had happened constantly in the global range, such as the accident in the Beijing MiHong park in 2004 that led to 37 people dead and 15 people being injured, about 362 dead in the Hajj in 2006; in 2008, a temple of Indian Rajasthan happened trample tragedy, 147 dead and 55 being injured; in the electronic music carnival of the German city of Duisburg, about 21 dead and 500 being injured in 2010 . The appearance of these calamities has made people pay more attention to the safety of public activities. Presently, China is in the period of society crisis: the population of city increases rapidly, the social activities' organizations are frequent, and the safety problems are urgently needed to be solved for the development of cities.
Accordingly, it is a critical subject to grasp the characteristics of occupant's crowding and evacuation and to find a more scientific people evacuation mode, which can help us seek new approaches of reducing the risk of emergency.

The pedestrian movement is more complex than vehicular flow for the reason that people are more flexible and intelligent than cars. Without the limit of "lanes," pedestrian movement is loose and free. Furthermore, the walkers are easily affected by others as well as the environment around, so the mutual interactions between people in the public places are regular, and they include homoplasy and repulsion [1]. Take the following action of pedestrian as an example: it means that the latter pedestrian walks following the former one with a uniform speed and route. When a person walks in a crowd, he must keep a sufferable distance from surrounding people as much as possible in order to avoid mutual impact because it makes people psychologically uncomfortable and makes the walking speed slower. The differences between people and cars are that people's collisions are permissible and vehicles' are not. In addition, the movement of people 
is also influenced by surrounding environment, lanes' conditions, and emergencies. All of these local and individual interactions, environmental and lanes' factors, and personal properties codetermine the pedestrian flow.

Recently, considerable research has been done on the study of pedestrian flow and has achieved a lot of constructive inclusions. The existing models studying human movement, including pedestrian flow and occupant evacuation, can be classified into two categories.

One is the microcosmic discrete model based on the traffic flow. The most widely used discrete models are CA and Gas-kinetic models [2, 3]. In [4-7], Nagatani et al. used Gas-kinetic model for some certain building structures like the large hall, bottleneck channel, and T-shaped channel to study how the structures influence the evacuation efficiency. Schadschneider et al. [8] provide an extensive overview of various aspects of pedestrian dynamics, focusing on evacuation processes, which reviews empirical results, discusses many modeling especially on cellular automata models, and presents some specific applications to safety analysis in public buildings. Reference [9] puts forward a new CA model which introduced the friction, and the repulsion considering the mutual function of people-people and people-environment. The authors of [10] only defined a probability function for route selecting and analyzed them, but the detailed mathematic equation of attraction and repulsion and their coefficients were not given. In [9], the attraction, the friction and the repulsion were added in their model, while the attraction produced by the motion directions of the ambient walkers was not considered, and the applicability for the high density crowding needs to be discussed since the walkers' routes selecting is not determined by individual's will but depends on the direction of pedestrian flows. Kirchner and Schadschneider [11] present simulations of evacuation processes using a recently introduced cellular automaton model for pedestrian dynamics which applies a bionics approach to describe the interaction between the pedestrians using ideas from chemotaxis. Helbing et al. [12] performed experiments for corridors, bottleneck areas, and intersections to test simulation models of pedestrian flows, and the result shows that the geometric boundary conditions are not only relevant for the capacity of the elements of pedestrian facilities but they also influence the time gap distribution of pedestrians, indicating the existence of self-organization phenomena.

While the other continuous flow model is based on the fluid dynamics, the most famous continuous model is the social forces model which is proposed by Helbing et al. $[13,14]$ and focused on the interaction of people-people and people-environment. Yang et al. $[10,15]$ present an occupant evacuation and movement CA model and study the selforganization phenomena and phrase transition. Burstedde et al. [16] propose a two-dimensional cellular automaton model to simulate pedestrian traffic and show that the introduction of such a floor field is sufficient to model collective effects and self-organization encountered in pedestrian dynamics, for example, lane formation in counter flow through a large corridor. Wen et al. [17] proposed a pedestrian evacuation model which combined the motion mode of the robot and the theory of CA. A modified dynamic cellular automata model is proposed to simulate the evacuation of occupants from a room with obstacles which takes into account some factors that play an important role in an evacuation process, such as human emotions and crowd density around the exits [18]. Cao and Shan [19] propose a new method to estimate the stock return and use boredom distribution to denote the final stock return which can reflect the features of technical patterns and investors' heterogeneity simultaneously. Gauthier [20] introduces a new class of derivative products that can hedge this risk which considers the hedging of the risk related to the cost of suboptimal entry or exit.

In a word, most of the research made use of the social forces model and the CA model separately. Although $[9,10]$ integrated the advantages of the two kinds of models, it was not perfect to some extent. In this paper, we introduce a new CA model for pedestrian evacuation and simulate the evacuation process of a plaza with one exit and a bidirectional channel, and we also presume that a trample accident happens in the process when the density of crowd is in a high level. After setting the isolated belt in the exit and the entrances of the channel, the causality in the accident sharply decreases.

\section{Establishment of the CA Model}

2.1. Hypothesis. The model in this paper is described in the two-dimensional plane system. The underlying structure is a $L \times L$ cell grid, where $L$ is the system size. Each cell can either be empty or occupied by exactly one pedestrian. The size of a cell corresponds to approximately $1 / 3 \mathrm{~m} \times 1 / 3 \mathrm{~m}$. This space takes the applicability of the model for the high density crowd seriously. According to [19], the human's body can show the deformation and compression because of the pressure; the density of the crowd is likely to reach 8 persons per square meter in limited cases. In [20], the most tolerable density of our country's crowd is 9 persons per square meter at an extreme situation after a precise calculation. That is the reason why most models do not accurately simulate the evacuation process.

The neighborhood selection of CA is pivotal as each cell's state depends on other cells in its field. The Moore type neighborhood is adapted in our model and its radius is two cells. The motion direction of pedestrian in Figure 1 shows that there are forward walkers moving from their own cells to the upper cells, the top right walkers moving to top right cells, and so on. Empirically, the average velocity of a forward pedestrian is about $1 \mathrm{~m} / \mathrm{s}$; the speed of the right and left walkers is also $1 \mathrm{~m} / \mathrm{s}$ while the backward walkers' is $1 \mathrm{~m} / \mathrm{s}$ and the others' is $\sqrt{2} \mathrm{~m} / \mathrm{s}$.

2.2. Update Rules. The update is synchronous for all pedestrians; each pedestrian can move only one cell per time-step. In the update, two problems, that is, the route choice and the confliction occurring when more than one pedestrian vies for a cell, will be solved. Our model has presumed that a cell must be occupied by one person, so we adopt roulette choosing method to confirm who can move to the aim cell, and others stay in their own primary cells. 


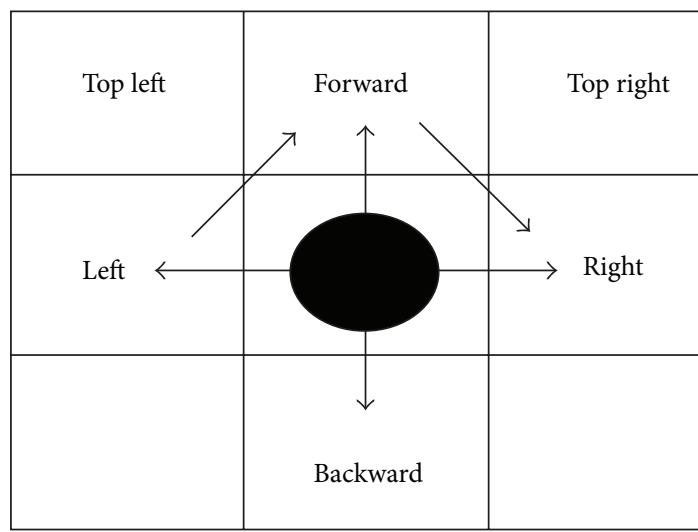

FIGURE 1: The motion direction of walkers.

The route choice is a significant issue, in our model, we introduce the approximate concept of the social forces and take the exit attraction, the repulsion, the friction, the repulsion force between human and barrier, and the attraction of surrounding people as objects codetermin the route choice. The definitions are as follows.

Firstly, the concept of the "floor field" is described to show the exit attraction, that is, each occupant is given a grid map, on which each cell has a degree of attractive force. For example, the exits have the greatest degree of attractive force during emergency evacuation. The math expression below is defined:

$$
E= \begin{cases}E_{0} & \exp \left\{-\min \left[\sqrt{\left(x_{i}-x_{0}^{m}\right)^{2}}+\sqrt{\left(y_{i}-y_{0}^{m}\right)^{2}}\right]\right\}, \\ \left(x_{i}, y_{i}\right) & \text { is not barrier } \\ 0, & \text { is barrier, }\end{cases}
$$

where $E_{0}$ is the maximum of the attraction, $\left(x_{i j}, y_{i j}\right)$ expresses the coordinates of the cell $(i, j),\left(x_{0}^{m}, y_{0}^{m}\right)$ is the coordinates of exits, and the total number of exits is $m$.

The repulsive force between people-people and peoplebarrier is introduced when the distance of people-people or people-barrier (like wall) reaches a little value; at this time, in order to avoid the collision and have a enough free space, walkers stay away from other walkers and barriers. The formula of the repulsions is

$$
R_{i j}(t)=A \exp \left[\frac{r_{i j}-d_{i j}}{B}\right],
$$

where $A$ indicates the interactive intensity, $B$ expresses the range of repulsion, $r_{i j}$ denotes the radius sum of two people and $d_{i j}$ shows the distance between two people or peoplebarrier. Besides, we define a repulsive coefficient to embody the different collision effects since the dissimilar collision can result in a different damage. That is,

$$
P_{r}=\frac{1-e^{-\alpha}}{1+e^{-\alpha}}
$$

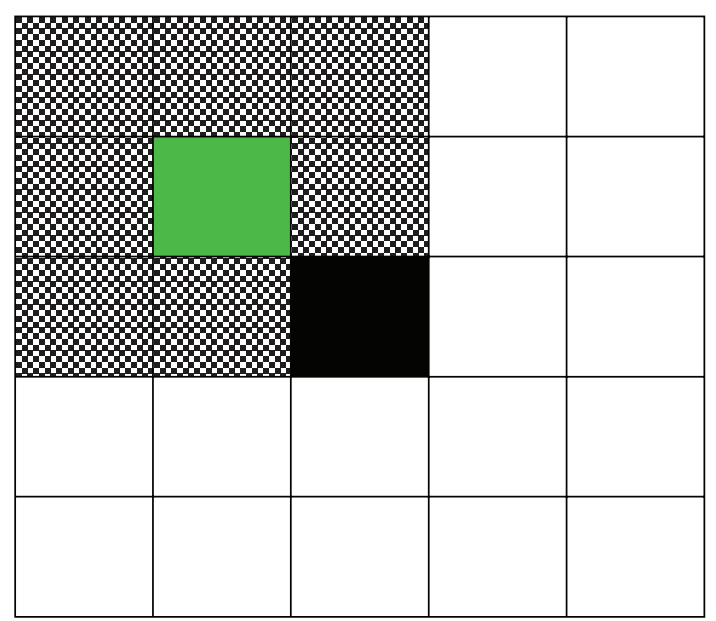

FIgURE 2: The possible calculating neighbors of an occupant.

where $\alpha \in[0, \infty]$ is rigidity coefficient indicating the hurt degree caused by barriers and people.

The friction between two occupants or between occupant and barrier is considered when the distance between them is too small because the influence of friction is often much stronger than the repulsive forces' in a high density. The simple equation is given as

$$
F_{i j(z)}=k \theta \varphi(z),
$$

where $k$ is a constant and $\alpha$ is rigidity coefficient; consider the following:

$$
\varphi(z)=\left\{\begin{array}{ll}
z, & z \geq 0, \\
o, & z<0,
\end{array} \quad z=r_{i j}-d_{i j}, \quad \text { if } z \geqslant 0,\right.
$$

that is to say, the sum distance between two occupants is larger than the sum radius between them, and two occupants collide, if $z<0$, that is the value is zero; $\theta$ is friction coefficient according to the impacted objects' roughness.

The occupant-direction-attractive force $D_{i j}$ : in the process of evacuation, the "follow" phenomena are often observed, which means that occupants tend to follow the movement of the majority. $D_{i j}$ describes the influence of the direction that most occupants have taken around the cell. In the model, the walkers may choose the same moving direction as most people have taken, this force is defined as

$$
D_{i j}=\beta N^{2},
$$

where $\beta$ is the influencing factor and $N$ is the total number of people within his neighborhood who choose the same moving direction.

Through analyzing the forces, the final following expression is defined to identify the occupant route choice:

$$
T_{i j}=\exp \left(-R_{i j}\right) \exp \left(-F_{i j}\right) \exp \left(D_{i j}\right) E .
$$

Considering that the friction and the repulsion have a hindering effect to walker's movement, while the exit 


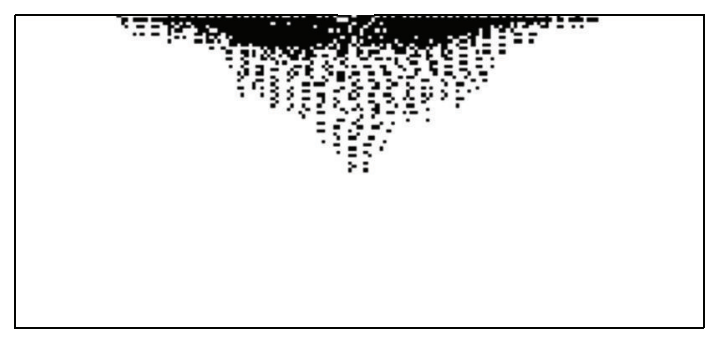

(a)

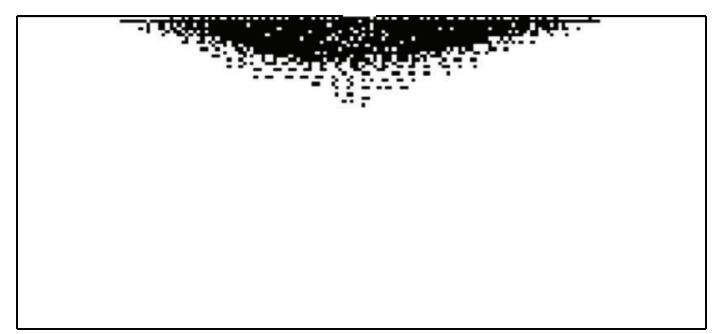

(b)

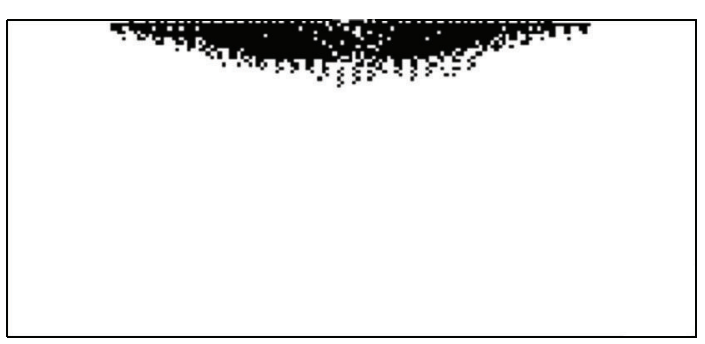

(c)

FIGURE 3: Simulations of evacuation in a stadium.

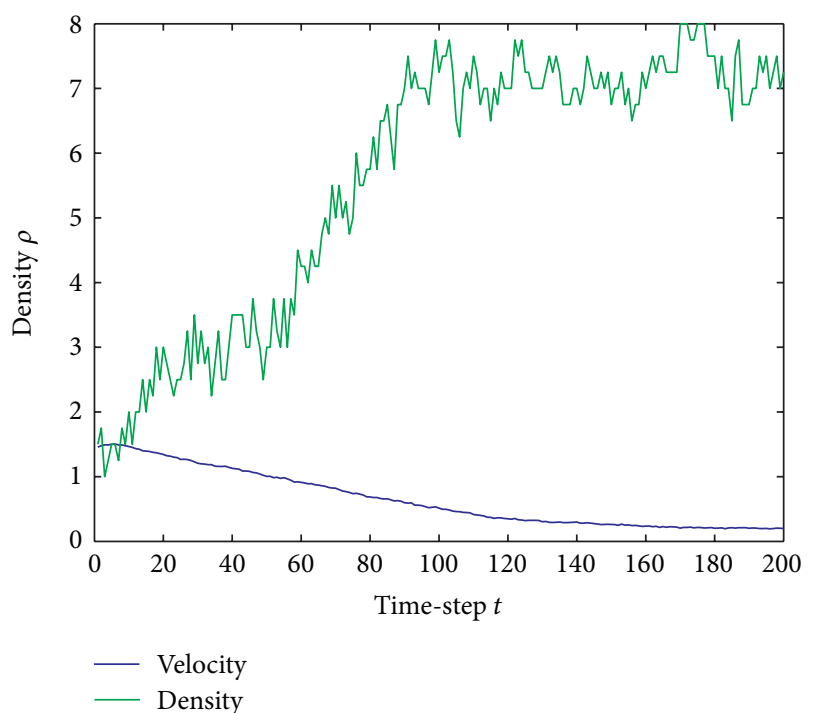

FIGURE 4: Velocity-time relation and density-time relation.

attraction and the occupant-direction- attractive force are beneficial to movement, $R_{i j}$ and $F_{i j}$ are negative and $D_{i j}$ and $E$ are positive. When a man chooses his route, the adjacent grid with a maximum attraction $T_{i j}$ is selected as his next timestep aim grid because it is the most favorable one for him.

Figure 2 shows the potential aim grids and calculating neighbors; the black grid indicates a man, the green grid is one of his possible aim grids for next time-step; if it is unoccupied, we view it as the core and calculate its attraction value $T_{i j}$ according to his seven neighbors except the black; otherwise, the value $T_{i j}$ equals zero. If a possible grid's value $T_{i j}$ is a maximum compared with other possible neighbors described in Figure 1, it is chosen as its aim grid.
In a word, the update rules are summarized as follows:

(1) each occupant chooses another cell near him as his aim grid, according to the state of his neighborhood and the route-choice rules;

(2) because one cell can only be occupied by one person at a time-step in our model, the conflict occurs when more than one person vies for a cell. In this situation, we adopt roulette choosing method to confirm who can move to the aim cell. The others have to stay at their origin position;

(3) after arriving at aim cell, the occupant will identify a new attractive-force-degree distribution on his map based on new environment and select his next aim cell;

(4) the update is synchronous for all occupants, when all updates end and turn to (1) for the next update.

\section{Simulations and Results}

3.1. The Simulation of Evacuation for a Stadium. In the simulations, we assume a rectangular stadium with a sole exit, and its size is $60 \mathrm{~m} \times 30 \mathrm{~m}$; the width of the exit is $2.7 \mathrm{~m}$. The rigidity coefficient $\alpha=1$ (repulsion between people-people), $\alpha=2$ (repulsion between people-barrier), the friction coefficient $\theta=0.1$ (friction between peoplepeople), and $\theta=0.5$ (friction between people-barrier). There are 1200 initial walkers in the stadium, and they are distributed randomly on the square lattice. Figure 3 shows the simulations of the occupant evacuation process.

Figure 3 shows the typical process of evacuation, and their separate corresponding time-steps is $t=100,150$, and 200; the practical corresponding time is $t=33 \mathrm{~s}, 20 \mathrm{~s}$, and $67 \mathrm{~s}$. We can clearly observe the basic evacuation dynamics phenomena from the plots, including arching and stagnation 


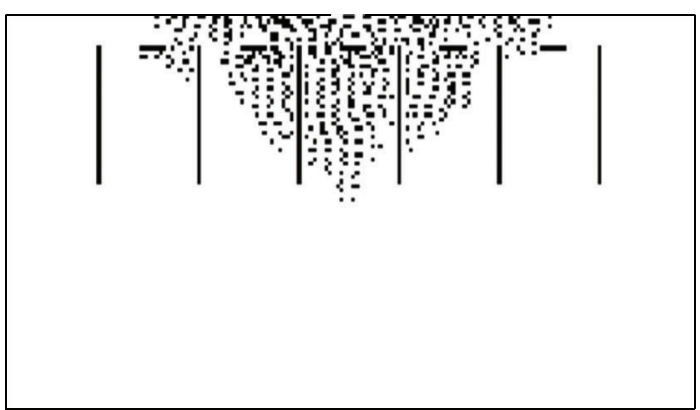

(a)

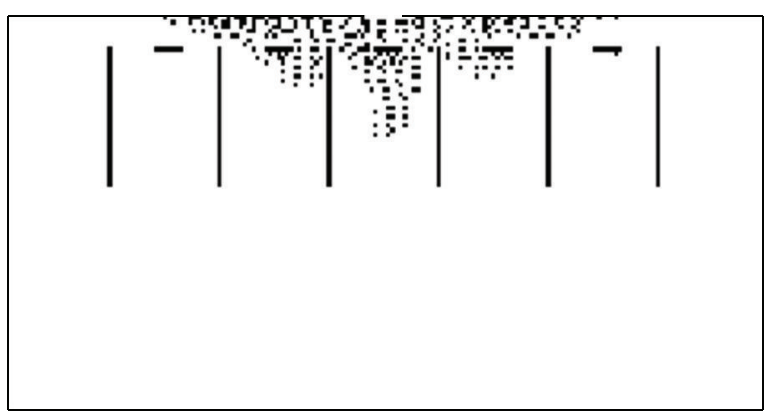

(b)

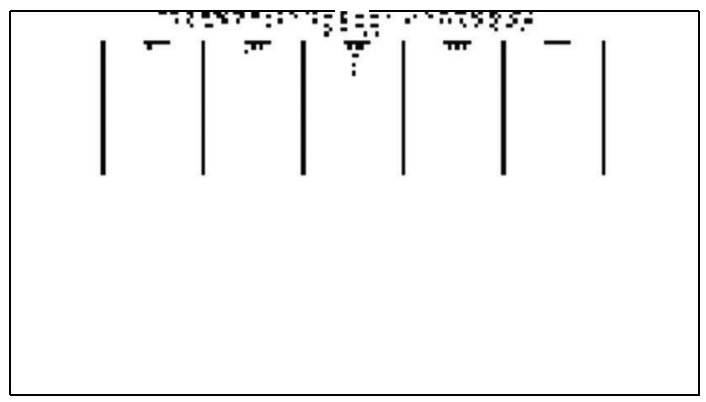

(c)

FIGURE 5: The simulation of evacuation process for a stadium setting the isolated belts.

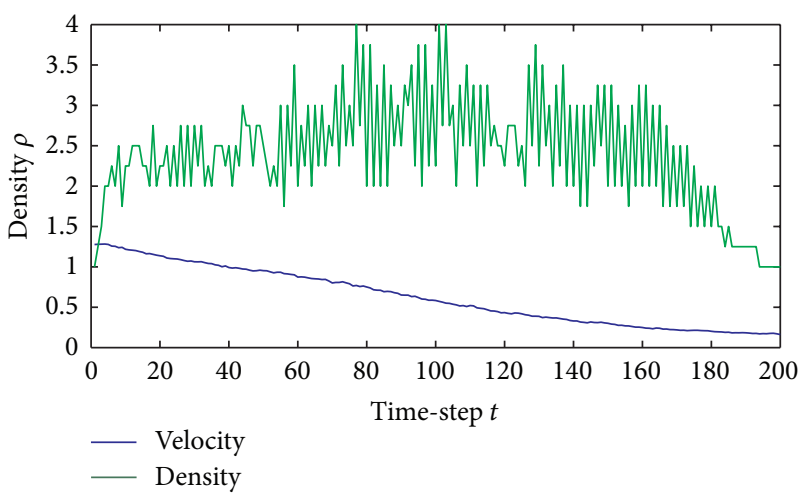

FIGURE 6: Velocity-time relation and density-time relation.

phenomenon; too many people assemble close to the door and form a serious blocking state, and there is too little space to move or even no free space to move, of course these are tallied with the actual situation.

Figure 4 shows the relation between walking velocity and time-steps and the relation between density and time-steps. The velocity is the average value that is calculated by the sum velocity of walkers in the range of $4 \mathrm{~m}^{2}$ nearby the exit. In Figure 4, at the starting moment, the average speed approaches the maximum $1.41 \mathrm{~m} / \mathrm{s}$; the density of crowd has a small value which is about $1.5 \mathrm{~m}^{2} /$ per man, so there is a large free space for pedestrians to walk. After a brief time, the density becomes higher and higher with the passage of time, which increases from about 0.67 people $/ \mathrm{m}^{2}$ to about
7 people $/ \mathrm{m}^{2}$, while, at this stage, the moving speed decreases gradually from the maximum to about $0.2 \mathrm{~m} / \mathrm{s}$, so we can conclude that the moving speed of a crowd depends on the crowd density, but not the individual speed, the higher crowd density, and the slower moving speed; when the density comes to the limit value (here it is 7 or 8 people per square meter), the moving speed comes to zero; this inclusion is consistent with [21].

In the above simulations, we find that the crowd density reaches 7 people $/ \mathrm{m}^{2}$, and its moving speed reaches $0.2 \mathrm{~m} / \mathrm{s}$. If people stay for a long time in a higher density crowd which will induce some physiological uncomfortableness, such as dyspnea, hypoxia of the heart and brain, and crowd panic, this uncomfortableness is easy to induce the trample disaster; therefore, we assume that a trample disaster happens nearby the exit once the density of crowd is over 6 people per square meter and the number of fatalities is noted at the same time. Through simulating the above evacuation using the same parameters, we get that the total number of casualties is 28 people at 100 time-steps, 71 people at 150 time-steps, and 122 people at 200 time-steps. According to this circumstance, some isolated belts are set to prevent the emergencies happening. The results are as follows.

Figure 5 shows the typical process of evacuation process, and their separate corresponding time-steps is similar to Figure 3. The isolated belts setting shows in the graphs, the distance between two vertical belts is $8 \mathrm{~m}$, and the distance between vertical belts and transverse belts is $2 \mathrm{~m}$ of walking. In Figure 5, we can also observe the arching phenomena, but there are some differences compared with Figure 4; obviously, the number of the rest of evacuation pedestrians is palpable 


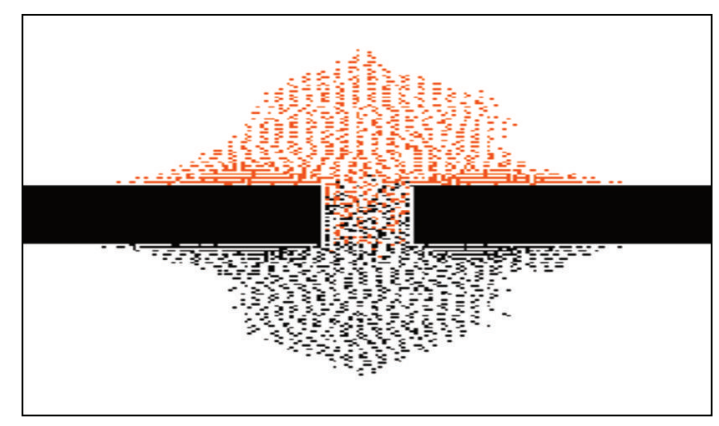

(a)

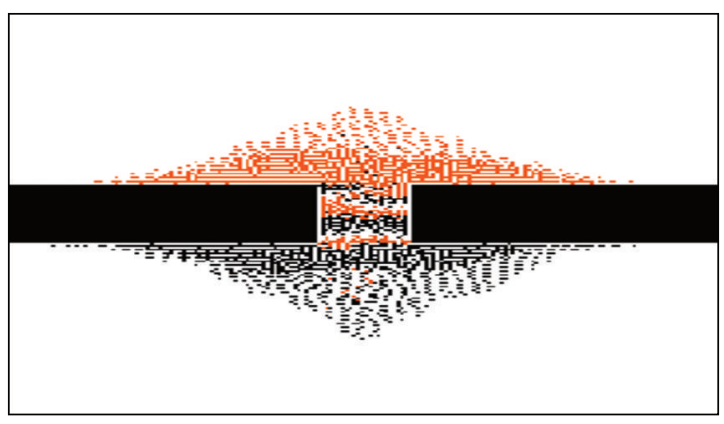

(b)

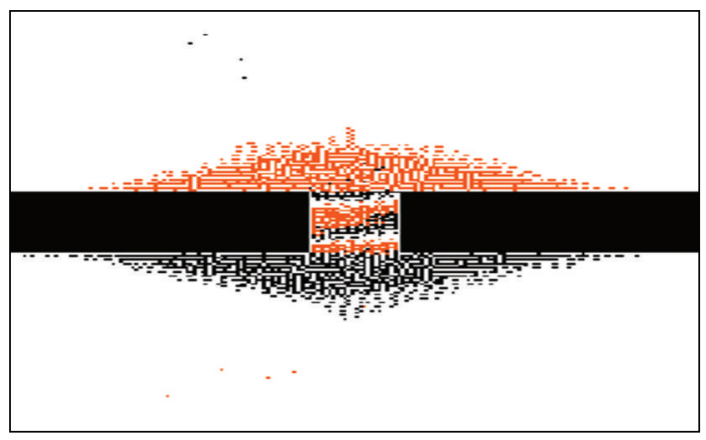

(c)

FIGURE 7: Simulations of evacuation for a bidirectional underpass.

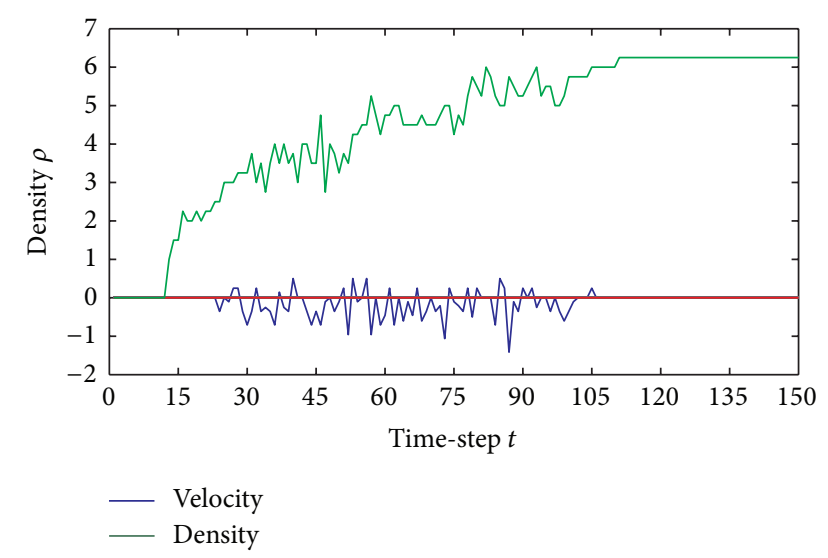

FIGURE 8: Velocity-time relation and density-time relation of simulation for the underpass.

fewer than that in Figure 3 at the same simulation time-steps, this sufficient improves that the moving speed is more faster.

Figure 6 shows the relation between walking velocity and time-steps and the relation between density and timesteps. In the graph, the density of crowd increases rapidly to $2-2.5$ people $/ \mathrm{m}^{2}$ with the passage of time, and the speed gradually decreases to $1.3 \mathrm{~m} / \mathrm{s}$. With the further passage of time, the density does not increase greatly and its value stays at 3 people $/ \mathrm{m}^{2}$ for a long time until 180 time-steps; from then on, the density starts to decrease because most people have evacuated and the people in the density statistical area descend. Form the analysis, we know that setting isolated belts can advance the evacuation efficiency substantially and decrease the crowd density, so the risk of accident happening is weakened to a great extent.

\subsection{The Simulation of Evacuation for a Bidirectional Under-} pass. This paper also simulates an evacuation process for a bidirectional underpass whose length is $10 \mathrm{~m}$ and width is $8 \mathrm{~m}$. At the initial moment, there are 1200 pedestrians distributed randomly on the bilateral 2-dimensional planes of the underpass.

Figure 7 shows the simulations of evacuation process for a bidirectional underpass, and their separate corresponding time-steps are $t=50,100$, and 150; the red grid points represent walkers who pass through the underpass from upper plane to under plane, while the black ones are just contrary. In Figure 7(a), pedestrians move to the entrances of the underpass at start evacuation moment, and form a typical arching, in Figure 7(b), the crowd density ascends furthermore, and "segregation phenomena" appears, and a steady jam phase forms inside the underpass. We can see that people separate into three blocking layers, and a few people in the heterogeneous directional crowd have passed the underpass. The reasons of the "segregation phenomena" have two aspects, one is that the heterogeneous pedestrians have no consciousness to prevent collisions between other people; another is the following action which incurs more people to join the clogging. In Figure 7(c), the layer phenomenon is more obvious, and the more density of the crowd is, the more congestion is produced.

Figure 8 shows the velocity-relation and density-time relation of simulations for the underpass. At the start moment 


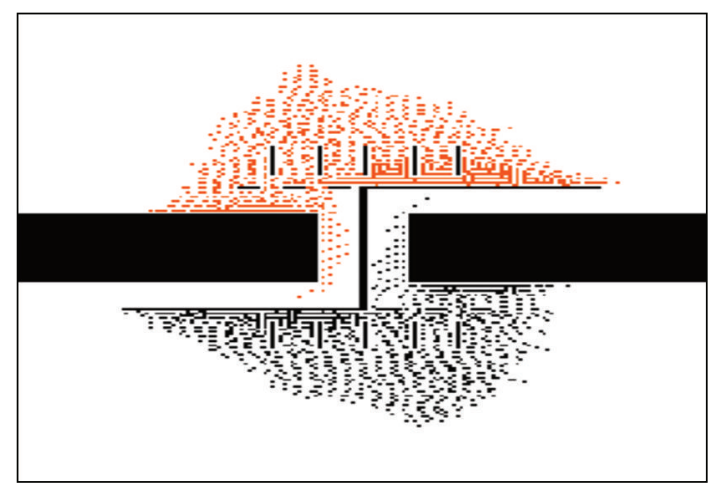

(a)

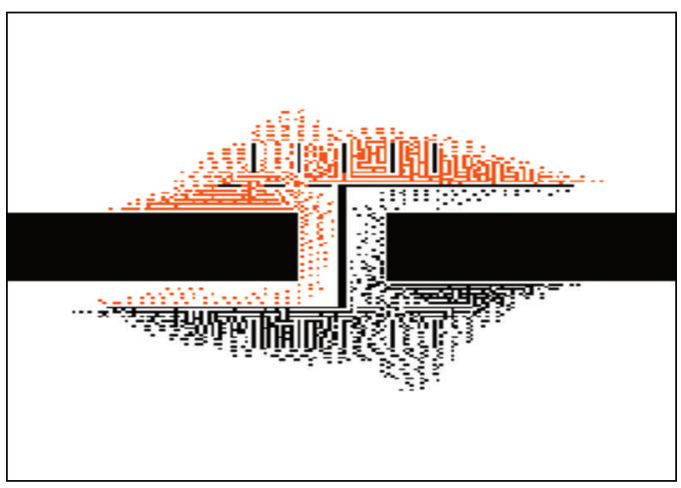

(b)

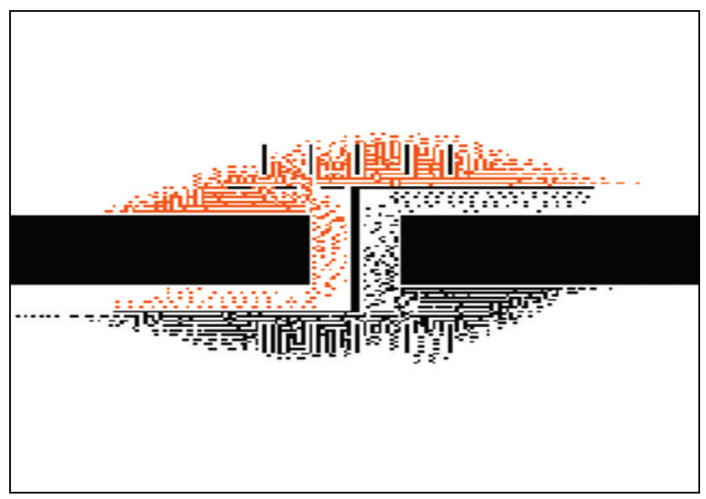

(c)

FIGURE 9: Simulations of evacuation for a bidirectional underpass after setting isolated belts.

of simulations, the density and the speed of crowd inside the underpass are zero, because the initial conditions assume that there are no pedestrians in the underpass. After a period, walkers move into the underpass, and the density of crowd ascends gradually; at the moment of 110 time-steps, the density stays at 6.2 people $/ \mathrm{m}^{2}$ and stays in this value for the rest of the time. The coming of this state is there is a steady "layer blocking" has formed and the walkers have to stay in their original positions. We see from Figure 8 that the density increases with the increase of time-step, and the velocity has the same trend. However, the variation of speed at the density of 4.5 people $/ \mathrm{m}^{2}$ is lager, which will increase the risk of conflict and interference between pedestrians. So, in the simulation, we presume that a crowding accident happens when the density is over 4.5 people $/ \mathrm{m}^{2}$; the number of casualties are 0,3 ; and 4 people at the time-steps 50 , 100 , and 150. We assume that the walkers' speed of red grid points is negative, while the walkers' speed of black ones is positive, so the sum of these two speeds counteracts each other and very small. At the start moment, the speed is zero because there is no one in the underpass. After a period, the walkers move into the underpass, but the density is still very small, and the summary speed counteracts to zero. When the density increases to about 2 people $/ \mathrm{m}^{2}$, the heterogeneous directional walkers start to interact and the mutual avoiding actions and surpassing actions appear, which lead to the speed fluctuated at zero. After the timesteps of 110, the speed stays at zero because of the blocking phase. The tramper disaster happened in the electronic music carnival of the German city of Duisburg which was similar to our simulations.

In order to prevent the occurrence of blocking phrase, we also set the isolated belts at the entrances of the underpass.

Figure 9 shows the pedestrians' evacuation process of the bidirectional underpass; the parameters are similar to the above instance. The setting of isolated belts displays in the plots; the full isolated belt is placed at the center of the tunnel; we design that all pedestrians walk by the right then turn right after passing the underpass and leave the system. In the figures, we can see a lower density outside the tunnel compared with Figure 7 as the isolated belts restrict the movements of walkers; the density of people inside the tunnel is more lower and there is no disturbance for bidirectional walkers; therefore, the number of walkers passing the tunnel is much more than the number of isolated belts, and the evacuation efficiency is advanced greatly.

\section{Conclusions}

In this paper, a cellular automata model is presented to simulate the two-dimensional pedestrian movement. Based on the characteristic of pedestrian movement and the social 
forces model, we have established a set of rules for route choice in pedestrian movement. The differences compared with previous research are that five kinds of "forces" are introduced to describe these effects, and the detailed expressions are given as well, analyzing the evacuation characteristics results of entrance and exit with isolated belts and without isolated belts. Using these behavioral rules and artificial instances, evacuation simulations is operated and emergency accidents are studied in our research. These simulations have improved the good applicability of our model and recurred the evacuation processes including crowding, blocking, and arching phenomena. In the simulations for a bidirectional underpass, the "layer blocking" phenomenon is found; it is a very important factor for crowding and clogging.

Besides, in order to prevent the occurrence of emergency for a high density crowd, we set the isolated belts for the exits and the entrances of the underpass and simulate the same instances; the results reveal that the setting of isolated belts can decrease the density of crowd and increase the evacuation efficiency remarkably. These conclusions are hopeful to monish the risk of crowd tramper disaster and prove some references for designer.

\section{Acknowledgments}

The authors wish to thank the references for his helpful comments and suggestions for improvement. This paper is supported by the Chinese State Social Science Fund Project (11CJY067), Chinese Ministry of Education Humanities and Social Sciences project (10YJA630126), and Natural Science Foundation of Gansu province (1107RJYA070, 1208RJZA164).

\section{References}

[1] J. J. Fruin, Pedestrian Planning and Design, Elevator World Inc., Mobile, Ala, USA, 2nd edition, 1987.

[2] V. J. Blue and J. L. Adler, "Cellular automata microsimulation for modeling bi-directional pedestrian walkways," Transportation Research B, vol. 35, no. 3, pp. 293-312, 2001.

[3] S. Hoogendoorn and P. H. L. Bovy, "Gas-kinetic modeling and simulation of pedestrian flows," Transportation Research Record, vol. 1710, pp. 28-36, 2000.

[4] Y. Tajima and T. Nagatani, "Clogging transition of pedestrian flow in T-shaped channel," Physica A, vol. 303, no. 1-2, pp. 239250, 2002.

[5] Y. Tajima, K. Takimoto, and T. Nagatani, "Scaling of pedestrian channel flow with a bottleneck," Physica A, vol. 294, no. 1-2, pp. 257-268, 2001.

[6] T. Nagatani, "Jamming transition in the traffic-flow model with two-level crossings," Physical Review E, vol. 48, no. 5, pp. 32903294, 1993.

[7] T. Nagatani, "Dynamical transition and scaling in a mean-field model of pedestrian flow at a bottleneck," Physica A, vol. 300, no. 3-4, pp. 558-566, 2001.

[8] A. Schadchneider, W. Klingsch, H. Klupfel, T. Kretz, C. Rogsch, and A. Seyfried, "Evacuation dynamics: empirical results, modeling and applications," in Encyclopedia of Complexity and Systems Science, pp. 3142-3176, Springer, Berlin, Germany, 2009.
[9] A. Kirchner, K. Nishinari, and A. Schadschneider, "Friction effects and clogging in a cellular automaton model for pedestrian dynamics," Physical Review E, vol. 67, no. 5, Article ID 056122, 10 pages, 2003.

[10] L. Yang, J. Li, D. Zhao, W. Fang, and W. Fan, "A microcosmic discrete occupant evacuation model based on individual characteristics," Science in China. Series E, vol. 47, no. 5, pp. 608-615, 2004.

[11] A. Kirchner and A. Schadschneider, "Simulation of evacuation processes using a bionics-inspired cellular automaton model for pedestrian dynamics," Physica A, vol. 312, no. 1-2, pp. 260-276, 2002.

[12] D. Helbing, L. Buzna, A. Johansson, and T. Werner, "Selforganized pedestrian crowd dynamics: experiments, simulations, and design solutions," Transportation Science, vol. 39, no. 1, pp. 1-24, 2005.

[13] D. Helbing and P. Molnár, "Social force model for pedestrian dynamics," Physical Review E, vol. 51, no. 5, pp. 4282-4286, 1995.

[14] D. Helbing, I. Farkas, and T. Vicsek, "Simulating dynamical features of escape panic," Nature, vol. 407, no. 6803, pp. 487490, 2000.

[15] L. Yang, W. Fang, J. Li, R. Huang, and W. Fan, "Cellular automata pedestrian movement model considering human behavior," Chinese Science Bulletin, vol. 48, no. 16, pp. 1695-1699, 2003.

[16] C. Burstedde, K. Klauck, A. Schadschneider, and J. Zittartz, "Simulation of pedestrian dynamics using a two-dimensional cellular automaton," Physica A, vol. 295, no. 3-4, pp. 507-525, 2001.

[17] W. G. Wen, H. Y. Yuan, and W. C. Fan, "A cellular automaton model for pedestrian dynamics based on the behavior of robot," Chinese Science Bulletin, vol. 51, no. 23, pp. 2818-2822, 2006.

[18] L. E. Aik and T. W. Choon, "Simulating evacuations with obstacles using a modified dynamic cellular automata model," Journal of Applied Mathematics, vol. 2012, Article ID 765270, 17 pages, 2012.

[19] G. Cao and D. Shan, "The effect of exit strategy on optimal portfolio selection with birandom returns," Journal of Applied Mathematics, vol. 2013, Article ID 236579, 6 pages, 2013.

[20] L. Gauthier, "Hedging entry and exit decisions: activating and deactivating barrier options," Journal of Applied Mathematics and Decision Sciences, vol. 6, no. 1, pp. 51-70, 2002.

[21] Q. M. Hu, W. N. Fang, and Y. Q. Jia, “The simulation of crowding and clogging for pedestrian crowd dynamics," Science in China. Series E, vol. 39, pp. 1034-1038, 2009. 


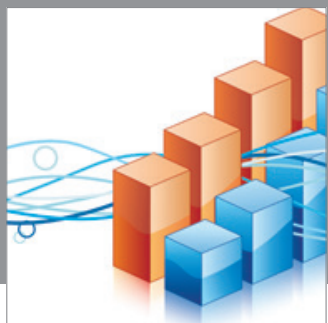

Advances in

Operations Research

mansans

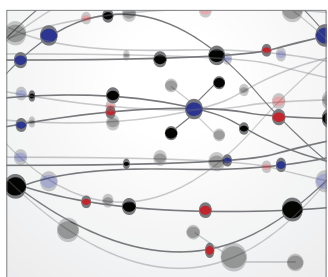

The Scientific World Journal
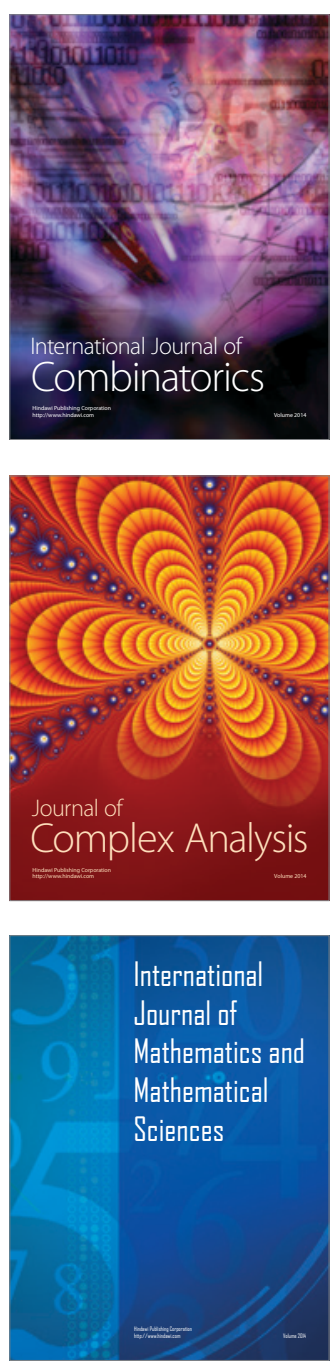
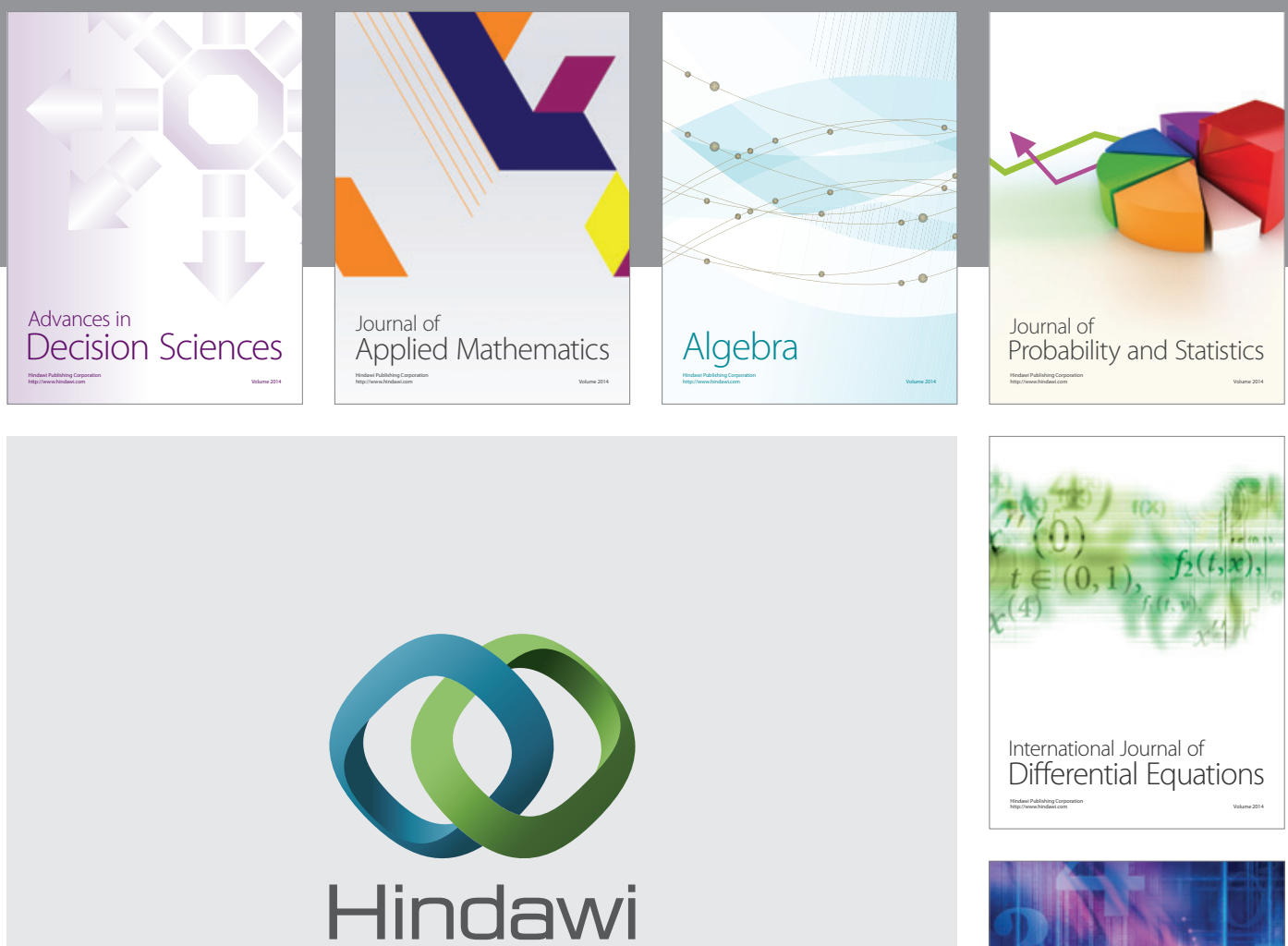

Submit your manuscripts at http://www.hindawi.com
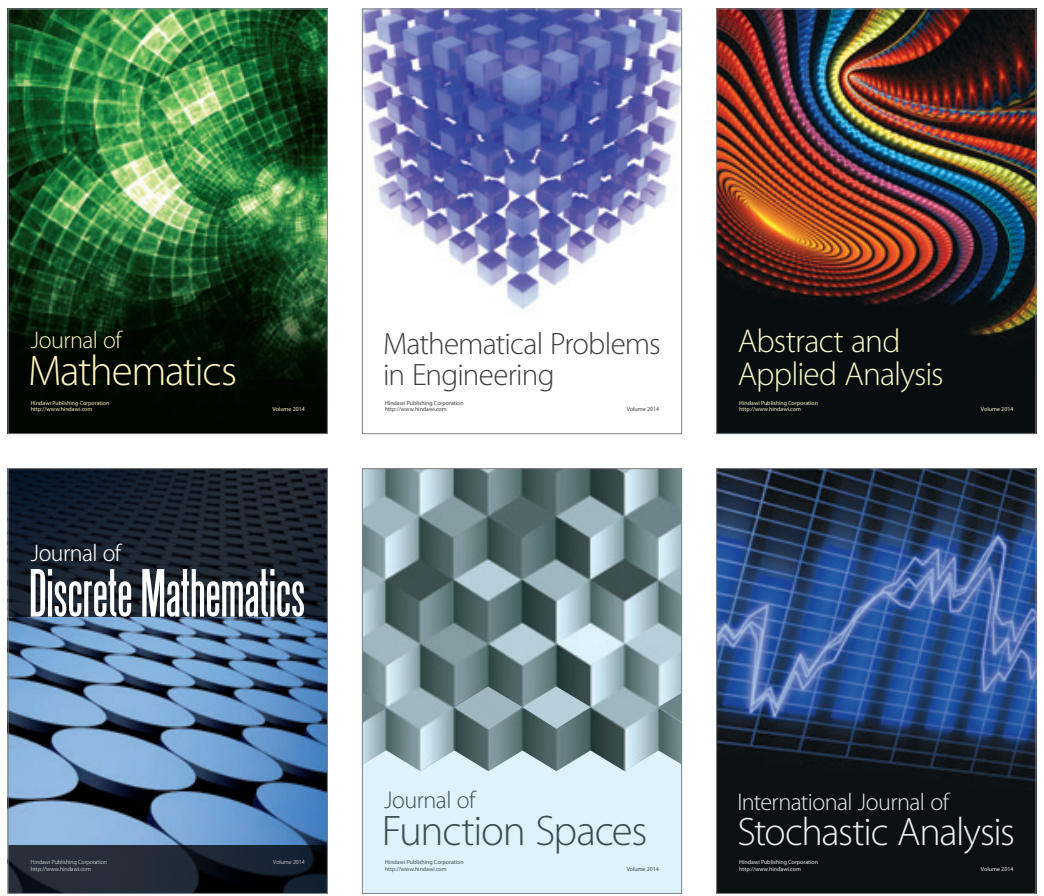

Journal of

Function Spaces

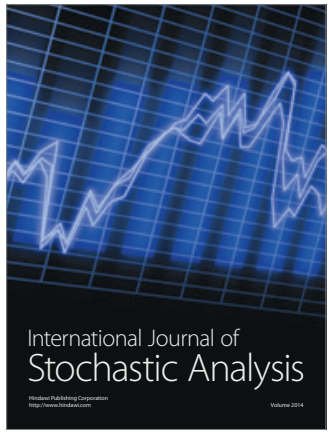

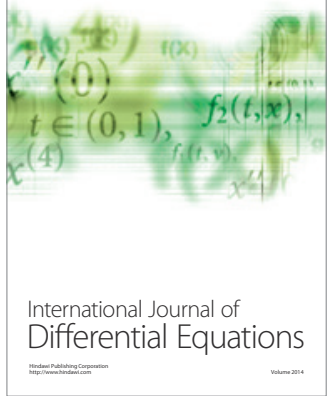
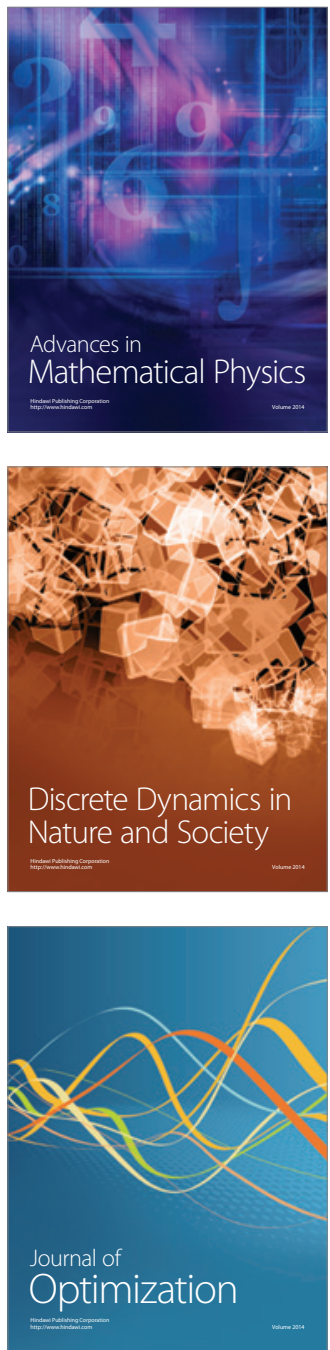